\title{
196 TLR9-ACTIVATED B CELLS DIRECTLY LICENSE ADOPTIVELY TRANSFERRED CD8+ T CELLS WITH POTENT TUMOR IMMUNITY
}

\footnotetext{
${ }^{1}$ Aubrey Smith*, ${ }^{3}$ Hannah Knochelmann, ${ }^{2}$ Megan Wyatt, ${ }^{2}$ Guillermo Rangel Rlvera, ${ }^{2}$ Brandon Ware, ${ }^{2}$ Amalia Rivera Reyes, ${ }^{3}$ Connor Dwyer, ${ }^{3}$ David Neskey, ${ }^{3}$ Mark Rubinstein, ${ }^{3}$ Bei Liu, ${ }^{3}$ Jessica Thaxton, ${ }^{4}$ Eric Bartee, ${ }^{2}$ Chrystal Paulos. ${ }^{1}$ Medical University of South Carolina, Charleston, SC, USA; Emory University, Decatur, GA, USA; ${ }^{2}$ Emory University, Decatur, GA, USA; ${ }^{3}$ Medical University of South Carolina, Charleston, SC, USA; ${ }^{4}$ University of New Mexico, Albuquerque, NM, USA
}

Background The use of immunotherapies such as immune checkpoint blockade or adoptive cell therapy has substantially improved outcomes for many patients with advanced malignancies. However, a majority of these patients still do not respond or relapse. Thus, extensive efforts are being made to improve these therapies. We and others have demonstrated that adoptive cell therapy can be improved by the co-administration of TLR agonists intratumorally. TLR agonists have also been administered to patients alongside a number of other immunotherapies, but often induce toxic side effects that may be exacerbated by cell therapy. We hypothesized that TLR agonists could alternatively be used in ex vivo cell culture to propagate a more potent cell therapy product.

Methods To address our question, we used a transgenic mouse model of tumor-infiltrating lymphocyte (TIL) therapy, pmel-1, in which CD8 $+\mathrm{T}$ cells express a TCR specific for a melanoma/melanocyte antigen. We activated CD $8+$ pmel- $1 \mathrm{~T}$ cells with APCs and the Toll-like receptor 9 agonist, CpG. Cell therapy efficacy was determined against mice bearing established B16F10 melanoma. Mechanisms underlying TLRimproved $\mathrm{T}$ cell products were determined using ex vivo cell depletion strategies and blocking antibodies.

Results CpG-expanded pmel-1 $\mathrm{T}$ cell products were much more effective against B16F10 melanoma in vivo than traditionally expanded T cells; they conferred more durable antitumor responses and improved survival of mice. CD8 $+\mathrm{T}$ cells generated in the presence of $\mathrm{CpG}$ also had heightened engraftment and persistence in the mice. We found that $\mathrm{CpG}$ did not act directly on $\mathrm{T}$ cells in culture, but on $\mathrm{B}$ cells, as depletion of B cells alone (not DCs, macrophages, NK cells, or CD4 cells) ablated the effects of CpG. B cells in CpGtreated cultures expressed a unique cytokine profile and upregulated several costimulatory markers on their cell surface, so we next questioned whether $\mathrm{CpG}$ improved the $\mathrm{B}$ cell/T cell axis via a direct or indirect (soluble) factor. Together, cell supernatant transfer experiments and costimulatory blockade experiments revealed that the direct interaction between $\mathrm{B}$ and $\mathrm{T}$ cells was required for the $\mathrm{CpG}$-mediated improvement of the $\mathrm{T}$ cell product.

Conclusions Our findings reveal a novel role for B cells in the generation of potent CD8 $+\mathrm{T}$ cell therapies against an aggressive solid tumor. These findings highlight a unique way $\mathrm{B}$ cells can become powerful APCs for generating effective antitumor $\mathrm{CD} 8+\mathrm{T}$ cells and can be directly translated to improve cell therapy products for patients with advanced malignancies.

Ethics Approval All animal procedures performed at the Medical University of South Carolina or Emory University were approved by each university's Institutional Animal Care \& Use Committee, protocol number 0488 or 201900225, respectively.

http://dx.doi.org/10.1136/jitc-2021-SITC2021.196 